\title{
EDITORIAL
}

\section{Reproducibility in Science}

\author{
Mauro Bertotti (iD $ه$ \\ Full Professor \\ Departamento de Química Fundamental, Instituto de Química, Universidade de São Paulo \\ São Paulo, SP, Brazil
}

Analytical chemists are particularly interested in measuring things, whether it is the amount of a substance or its concentration. When we develop a new method or technique to obtain such quantitative information, it must substantially improve the measurement conditions, perhaps by providing significant decreases in analysis times or by an enhancement in the analytical figures of merit. Like any other scientist, we produce results, and they have to be reproducible and reliable. Hence, an experiment should be described in such a way that other researchers with sufficient skills and resources can follow the steps and obtain identical results, within the margins of experimental error.

The reproducibility in an experiment is the foundation of the scientific method, and we have to validate the results at every stage of discovery and development, i.e., we have to be able to reproduce the work with precisely the same output. Reproducibility testing offers us the ability to deal with different parameters that can influence measurement results and estimated uncertainty. This is crucial because if we know the parameters that significantly impact measurements, we can take action to control the measurement process and reduce uncertainty in the results.

When it comes to reproducibility testing, there are plenty of different conditions that can be evaluated, and a distinction regarding the concept of "repeatability" should be introduced. Measurement repeatability states the closeness of the results obtained with the same sample using the same measurement procedure, same operators, same equipment, same operating conditions, and the same laboratory over a short period. The short period is typically one day or one analytical run. On the other hand, "reproducibility" expresses the precision between measurement results obtained at different laboratories (also named between-lab reproducibility) or the precision obtained in the same laboratory over a more extended time. Reproducibility considers more changes than repeatability, such as different analysts, operators, or instruments, and these parameters act randomly in the context of precision. Hence, because more variables are accounted for in the assessment of reproducibility, its value (expressed as the standard deviation) is larger than the repeatability. Reproducibility can be best explained as the standard deviation of multiple repeatability tests carried out under different experimental conditions.

Communicating a scientific result requires enumerating, recording, and reporting all experimental conditions to attempt repetition straightforwardly. However, several reports have suggested severe issues with the reproducibility of scientific research. Results that are not reproducible are unsound or derived from flawed experimental methods, or there is dishonesty in reporting the measurements. In a scenario in which science is questioned, such lack of rigor may be used against scientists, and, not surprisingly, concerns have been expressed in both scientific and popular media. To overcome such a problem, especially in the field of Analytical Chemistry, a possibility we might want to take into account is based on increased attention to statistics and data analysis, as well as using larger sample sizes. In a broader context, researchers and academic institutions should employ all efforts to improve reproducibility in science.

Cite: Bertotti, M. Reproducibility in Science. Braz. J. Anal. Chem., 2020, 7 (26), pp 2-3. doi: http://dx.doi.org/10.30744/ brjac.2179-3425.editorial.mbertotti.N26 


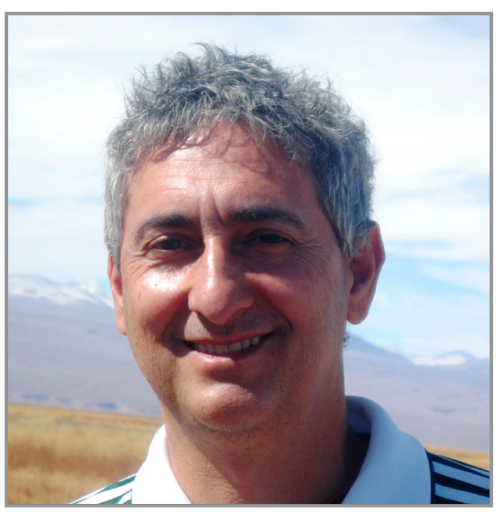

Mauro Bertotti graduated from the Institute of Chemistry at the University of São Paulo (IQ-USP), Brazil, with a degree in chemistry (1983), and holds a master's degree (1986) and a doctoral degree (1992) from the same IQ-USP and a postdoctoral degree from the University of Southampton (UK) (1995-1996).

$\mathrm{He}$ is currently a Full Professor at the IQ-USP and works mainly on electroanalytical chemistry, with emphasis on microelectrodes, modified electrodes, scanning electrochemical microscopy, and miniaturized and integrated chemical sensors as new manufacturing platforms for biological, clinical and environmental applications.

CV: http://lattes.cnpq.br/3497889001562167

p ResearcherID 